\title{
Alles auf den Kopf stellen
}

\author{
Zur besonderen Qualität interkulturellen Lernens im bilingualen \\ Sachfachunterricht ,Darstellendes Spiel‘
}

\section{Heike Wedel}

\begin{abstract}
Zusammenfassung
In den vergangenen Jahren hat sich der bilinguale Sachfachunterricht zu einem immer beliebter werdenden Angebot besonders an deutschen Gymnasien entwickelt. Dabei gibt es große Unterschiede in der Organisation (vgl. Hallet $2005 \mathrm{zu}$ Organisationsformen), in der lokalen Verbreitung und in der Verteilung auf die unterschiedlichen Schulformen, Schulstufen, Schulfächer und Sprachen (vgl. Werner 2007 mit aktuellen Zahlen). Traditionell überwiegen die englischsprachigen Angebote in Verbindung mit gesellschaftswissenschaftlichen Fächern. Das Unterrichtsfach ,Darstellendes Spiel' wird bisher sowohl von Seiten der Bildungspolitik als auch in der Theorie und in der Praxis des bilingualen Unterrichts kaum berücksichtigt. Das verwundert angesichts der vielen Schnittmengen zwischen Theaterspielen und Spracherwerb sowie der vielbeschworenen Potenziale im Hinblick auf das interkulturelle Lernen durch Theaterspielen. Im folgenden Beitrag sollen die Chancen und Besonderheiten des interkulturellen Lernens im bilingualen Sachfachunterricht ,Darstellendes Spiel' auf der Grundlage von Ansätzen aus dem Fremdsprachenunterricht, der Theaterpädagogik und der Dramapädagogik am Beispiel des Englischunterrichts dargestellt und diskutiert werden.
\end{abstract}

\section{Einleitung}

Immer häufiger wird zum Erreichen der komplexen und anspruchsvollen interkulturellen Lernziele im Fremdsprachenunterricht und in der interkulturellen Pädagogik sowie Theaterarbeit allgemein auf Techniken und Verfahren aus theater- und spielpädagogischen Arbeitsfeldern oder der Schauspielerausbildung verwiesen. Im Rahmen interkultureller Pädagogik wird Theaterarbeit zum Beispiel dazu eingesetzt, es den Beteiligten zu ermöglichen, Konflikte zu klären und tabuisierte Themen verfremdet darzustellen. Das Theaterspielen eignet sich nach Auernheimer (2003) dazu, bestehende Hierarchien infrage zu stellen und sie für die Zeit der Aufführung außer Kraft zu setzen. Einen besonderen Blick auf andere Kulturkreise ermögliche die Einbeziehung ihrer spezifischen 
Theaterformen (z.B. italienische Commedia dell'arte ${ }^{1}$, japanisches Kabuki ${ }^{2}$; cf. ibid. 160 sowie Regus 2009 zu Theater als interkultureller Begegnung). In der aktuellen Diskussion um geeignete Wege zur Förderung der interkulturellen fremdsprachlichen Handlungsfähigkeit im Rahmen des Fremdsprachenunterrichts spielen Rollenspiele, Simulationen, Dramentexte, dramatische Formen der Textarbeit, Dramapädagogik ${ }^{3}$ und alle auf diese komplexen Verfahren vorbereitenden Übungen eine wichtige Rolle. Es wird argumentiert, dass diese Verfahren geeignet seien, Perspektivenwechsel anzubahnen.

In diesem Beitrag sollen Ansätze interkulturellen Lernens aus den Bereichen Fremdsprachenunterricht und Theaterpädagogik ${ }^{4}$ exemplarisch vorgestellt und diskutiert werden. Im Anschluss daran wird auf dieser Grundlage ein Ansatz interkulturellen Lernens für den bilingualen Sachfachunterricht ,Darstellendes Spiel' entwickelt, der fremdsprachendidaktische und theaterpädagogische Elemente integrieren soll.

\section{Interkulturelles Lernen mittels Theaterspiel - Darstellung unterschiedlicher Ansätze}

\subsection{Interkulturelles Lernen in der Fremdsprachendidaktik am Beispiel Englisch}

Im Folgenden soll die Stellung interkulturellen Lernens im Bereich des schulischen Fremdsprachenunterrichts exemplarisch am Beispiel der Berliner Rahmenplanvorgaben für das Fach Englisch skizziert werden. Die Berliner Rahmenplanvorgaben für das Fach Englisch in der Sekundarstufe I und II zielen u.a. auf die Entwicklung der interkulturellen Handlungsfähigkeit ab, die in die Bereiche Sprachkompetenz, Methodenkompetenz und kulturelle Kompetenz unterteilt wird. Die Förderung interkultureller Kompetenz beinhaltet neben der Aneignung landeskundlichen Faktenwissens über englischsprachige Länder, Lebensräume, Literaturen und Kulturen sowie der sprachlichen Vorbereitung auf interkulturelle Begegnungen auch die Förderung von Einstellungen gegenüber diesen Kulturen. Diese Einstellungen sollten idealerweise von Offenheit und

\footnotetext{
${ }^{1}$ Im 16. Jahrhundert entstandene italienische Stegreifkomödie, bei der nur der Grundriss der Handlung und einige Typen (Arlecchino, Pantalone, Colombina, Truffaldino und Capitano) feststehen (cf. Graham 1999: 138).

${ }^{2}$ Japanisches Theater, das auf volkstümliche Tänze zurückgeht und sich ab dem 17. Jahrhundert in Japan entwickelte (ibid. 59).

${ }^{3}$ Dramapädagogik wird in diesem Aufsatz in Anlehnung an Schewe (2007: 10) als ein Oberbegriff für ein breites Spektrum an prozess- und produktionsorientierten Unterrichtsmethoden verstanden, die die Bereiche Fremdsprachenvermittlung und Drama/Theater einschließen.

${ }^{4}$ Der Begriff ,Theaterpädagogik' dient vor allem als Sammelbegriff für die verschiedenen Tätigkeitsfelder von Theaterpädagogen: von der sprachlichen Förderung im Kindergarten über therapeutische und resozialisierende Bereiche, z.B. Gefängnsistheater, bis hin zum Coachen von Führungskräften in Unternehmen. Theaterpädagogik ist außerdem die Bezeichnung für eine Handlungswissenschaft an der Hochschule (cf. Worsch 1996: 1455ff.; Rademacher 2003: 29f.).
} 
Neugier geprägt sein und die SchülerInnen gleichzeitig ermutigen, Stereotype und Wertungen kritisch zu hinterfragen (cf. Senatsverwaltung 2005: 9).

Eine besondere didaktische und methodische Herausforderung stellt die Entwicklung der interkulturellen Kompetenz mit ihren Bereichen Einstellungen, Wissenserwerb und Perspektivübernahme dar. Es geht um die Befähigung der SchülerInnen zum kritischen Blick auf die eigene und die fremde Kultur. Eine wichtige Rolle spielt in diesem Prozess die Einnahme der Sichtweise des anderen auf die eigene Kultur sowie die stete Hinterfragung der eigenen Perspektive gegenüber einer fremden Kultur. An den Englischunterricht wird die Erwartung herangetragen, ein Bewusstsein für sprachliche und nichtsprachliche kulturelle Unterschiede zu entwickeln und Verhaltensweisen und Strategien zu fördern, die einen sinnvollen Umgang mit diesen Unterschieden ermöglichen (vgl. dazu Roche 2001). Dabei handelt es sich nach Raddatz (2002: 133) beim interkulturellen Lernen um einen Prozess, der nie ganz abgeschlossen sein kann.

Laut Caspari (2000: 84) erfolgt die Wahrnehmung, Deutung und Beurteilung von Fremdem im Fremdsprachenunterricht über eine Reihe von Teilzielen und -aktivitäten wie z.B. das Nachvollziehen, das Imaginieren, das Hineinversetzen, das Zeigen von Gefühlen, das Vergleichen, das Begründen, das Anwenden von Wissen und die Entwicklung von Empathie. Rollenspiele und Theaterformen hält Caspari zum Erreichen dieser Teilziele für besonders geeignet. Nünning \& Surkamp (2006: 146f.) sehen die Bedeutung dramatischer Formen der Textarbeit und von Dramentexten im Kontext eines auf interkulturelle Handlungsfähigkeit ausgerichteten Englischunterrichts beispielsweise in folgenden Aspekten:

- Die Unmittelbarkeit dramatischer Texte erleichtert Lernenden das Hineinversetzen in fremde Situationen und Kontexte.

- Dramatische Texte dienen der Erprobung der Übernahme einer fremden Perspektive, indem eine Einfühlung in eine fremde Erlebniswelt erfolgt.

- Szenisches Spiel setzt zum Verstehen fremder Kulturen die zeitweilige Aufgabe eigenkultureller Erfahrungen, Wahrnehmungskonzepte und Deutungsmuster voraus. Lernende begegnen im Spiel verschiedenen Wirklichkeitsansichten.

- Dramatische Texte fördern durch ihre gattungsspezifischen Besonderheiten den aktiven Perspektivenwechsel.

Bonnet \& Breidbach (2007: 268) halten in Bezug auf die Entwicklung einer reflexiven Didaktik und Methodik des Fremdsprachenunterrichts Rollenspiele und Simulationen für besonders relevant und erwähnen das Identifikationspotenzial dieser Aufgaben sowie die Möglichkeit des aufgabenorientierten Arbeitens. Starkes Engagement und emotionale Beteiligung im Rahmen von Projektarbeit entsprächen den Prinzipien reflexionstauglicher Inszenierungsformen.

Alle genannten fremdsprachendidaktischen Ansätze haben vor allem eines gemeinsam: Theaterspielen wird als eine besonders gute Möglichkeit verstanden, 
um sich in andere Personen und Verhältnisse einzufühlen und sich ggf. mit dem und den Dargestellten zu identifizieren. Es wird davon ausgegangen, dass ein Perspektivwechsel u.a. durch aktives Ausagieren vollzogen werden kann. Wie im dritten Abschnitt gezeigt werden soll, stellt diese Sicht auf das Theaterspielen als Identifikationspotenzial und Mittel zur Einfühlung in fremde Welten nur eine mögliche Einsatzweise dar, die noch dazu spezifische Schauspieltechniken erfordert. Für Laien und Lernende ohne schauspielerische Vorerfahrungen sowie für das interkulturelle Lernen im Unterricht gleichermaßen besser geeignet halte ich den Einsatz von Theaterspielen als Mittel zur Distanzierung und zur aufmerksamen Wahrnehmung von Unterschieden und Gemeinsamkeiten sowie als Anregung zu einer vertieften Reflexion über das Wahrgenommene. Diese Position soll im Folgenden am Beispiel des Theaterpädagogen Sting und am Beispiel des Berliner Lehrplans für das Unterrichtsfach ,Darstellendes Spiel ${ }^{\star}$ näher ausgeführt und im vierten Abschnitt als zentral für das bilinguale Lernen im Fach ,Darstellendes Spiel‘ bestimmt werden.

\subsection{Interkulturelles Lernen in der Theaterpädagogik und beim Darstellenden Spiel}

Die Dimension interkultureller Bildung ist gegenwärtig auch Gegenstand theaterpädagogischer Überlegungen. Oft geht es dabei um Theater mit MigrantInnen oder mit DarstellerInnen mit Migrationshintergrund, das sich durch seinen Performanzcharakter ${ }^{5}$ auszeichnet und fernab vom konventionellen Theaterbetrieb biographische Einblicke in Themen wie Heimat, Integration, Zugehörigkeit, Fremdheit oder Identität gewährt (cf. Sting 2008: 87 sowie zu Theaterarbeit mit jungen MigrantInnen cf. Hoffmann \& Klose 2008).

Sting (2008: 89) beobachtet folgende Formen und Haltungen von Interkulturalität in interkulturellen Theaterprojekten: Exotismus, Internationalität, Transkulturalität und Hybridkulturalität. Beim Exotismus interessiere nur die Exotik des Fremden, und es entstehe nichts Neues. Internationalität setzt Sting mit dem beziehungslosen Nebeneinander verschiedener Theaterformen gleich. Hybridkulturalität hingegen beziehe sich auf das Mit- und Nebeneinander verschiedener Kulturtraditionen und deren Vermischung und erzeuge eine Bandbreite unterschiedlicher Spielformen jenseits der Polaritäten von Ich und Anderen. Für Sting erscheint das Interkulturelle im theaterpädagogi-

\footnotetext{
${ }^{5}$ Mit der performativen Wende ist in Bezug auf das Theater eine Entgrenzung des Theaterverständnisses gemeint, welche in den 1960er Jahren ihren Anfang nahm und Theater mit folgenden Merkmalen hervorbringt: Ereignis statt Werk, Präsentation statt Repräsentation, Handeln statt Spielen, Selbstdarstellung statt Rollen- und Figurendarstellung, Zuschaueransprache statt vierte Wand und Illusion (cf. Sting 2008: 93). Vgl. auch den folgenden Kommentar von Sting (ibid.): „Entlastet von der narrativen Komplexität und Geschlossenheit einer Dramenvorlage steht bei performativen Eigenproduktionen die Spielaktion im Mittelpunkt. Das bedeutet allerdings keine Beliebigkeit der Form, sondern erfordert ein Konzept, das Körperlichkeit und Ausdrucksfähigkeit der Spieler, Spielort, szenische Handlung, Spielweise und thematischen Bezug zusammen denkt. Ästhetisches Lernen zeigt sich dann nicht auf der Ebene dramatischer Rollenarbeit, sondern auf der Ebene der reflektierten (Selbst-)Darstellung unter Einbeziehung biographischen Materials und der bewussten Gestaltung performativer Spielformen.“
} 
schen Bereich (hier von ihm definiert als Theaterarbeit mit Nichtprofis) auf inhaltlich-thematischer Ebene z.B. durch Themen wie Fremdheit, Rassismus und Gewalt oder auf sozialer und formal-ästhetischer Ebene durch spezifische Ausdrucksformen, die die SpielerInnen innerhalb des Gruppenkontexts und auf der Grundlage unterschiedlicher Sprachen und ethnischer Bezüge entwickeln (cf. ibid. 89f.).

Den besonderen Wert interkultureller Theaterarbeit sieht Sting darin, dass sie „über die Produktion von Bildern, Zeichen, Symbolen und Geschichten die Differenz zeigen, thematisieren, betonen und zuspitzen [kann], ohne vorschnell Wertungen zu treffen" (cf. ibid. 91). Darüber hinaus enthalte der Theaterprozess ein Moment der Unterhaltung, was Sting resümieren lässt, dass eine Differenzerfahrung, die nicht von Angst und Xenophobie begleitet sei, ein positives Kulturerlebnis darstelle. Das Besondere des künstlerischen Arbeitens bestehe gerade darin, das vom Alltag Differente hervorzuheben und das Irritierende ins Zentrum von Inszenierungen zu stellen, um darüber in einen Dialog zu kommen:

Im Kommunikationsraum Theater kann das Differente und Eigene gezeigt und ausgestellt werden. Theater vermittelt über das Zeigen von Differenz - das ist pädagogisch und politisch gleichermaßen bedeutend - individuelle Lernprozesse und öffentliche Kommunikation (Sting 2008: 91).

So könne man Theaterpädagogik auch als „eine Schule des Sehens, des differenzierten, neuen und anderen Sehens" bezeichnen und Theaterspielen als „ein subjektives, soziales, thematisches und gestalterisches Bewusstwerden und öffentliches ,Sprechen“", also als Prozesse, die die Beteiligten anregen können, den Blick auf sich und die Welt zu verändern (cf. ibid. 92).

Auch im schulischen Unterrichtsfach ,Darstellendes Spiel ${ }^{`}$ spielen das interkulturelle Lernen und die Reflexion über die im Spiel generierten Erfahrungen einewichtige Rolle. Exemplarisch soll der Berliner Rahmenlehrplan für die gymnasiale Oberstufe herangezogen werden, der in Bezug auf das interkulturelle Lernen im Fach z.B. ,Darstellendes Spiel' feststellt:

Darstellendes Spiel fördert die Fähigkeit, mit sozialer und kultureller Heterogenität angemessen und produktiv umzugehen. Die Schülerinnen und Schüler sind aufmerksam und offen für Perspektivenwechsel und Ambiguitätserfahrungen. Im Spiel thematisieren sie unter Zuhilfenahme ästhetischer Prozesse Herkunft, Tradition, Geschlecht, Sprache und andere differenzierende Merkmale und bearbeiten spielerisch ihre Erfahrungen mit dem Ich und dem Anderen bzw. dem Eigenen und dem Fremden. Darstellendes Spiel ermöglicht den Schülerinnen und Schülern aufgrund der reflektierten Auseinandersetzung mit der eigenen Rolle und Sichtweise die gleichen Zugangs- und Teilhabechancen. Sie lernen unterschiedliche Handlungsmuster kennen und akzeptieren (Senatsverwaltung Berlin 2006a: 9).

Gemeinsam ist den beiden hier beschriebenen Positionen, dass sie der Darstellung und Wahrnehmung von Differenz mit Hilfe von Theaterspiel sowie 
der Reflexion darüber einen zentralen Stellenwert einräumen. Damit setzen sie einen anderen Schwerpunkt als diejenigen Konzepte, die der Entwicklung der Fremdsprache durch das Theaterspiel den Vorrang geben.

\section{Interkulturelles Lernen mittels Theaterspiel - Diskussion unterschiedlicher Ansätze}

Wie im zweiten Abschnitt dieses Beitrags dargestellt, wird das Theaterspielen im Fremdsprachenunterricht vor allem als eine Möglichkeit angesehen, interkulturelles Orientierungswissen zu vermitteln und eine Einfühlung der Lernenden in eigene und fremde kulturelle Gegebenheiten anzubahnen. Voraussetzung dafür ist, dass das Theaterspielen als vor allem identifikationsstiftende Tätigkeit angesehen wird, die keine speziellen theaterästhetischen Voraussetzungen oder Kenntnisse erfordert, und dass angenommen wird, dass man zwischen eigener und fremder Kultur klar unterscheiden kann. Auf welcher Grundlage und wie genau diese Einfühlungs- und Identifikationsprozesse verlaufen sollen, wird in der Regel nicht im Detail erörtert. Nicht selten beruhen die angeführten Begründungen auf einer eher unreflektierten Übernahme einer bestimmten, historisch geprägten Vorstellung von Theater. Auch als Ausgangspunkt für Reflexionen kommt Theaterspielen in der Fremdsprachendidaktik zunehmend ins Spiel. Aber auch hier fehlt bisher die genaue Darstellung der Grundlagen für theaterbasierte Reflexionsprozesse.

In Ansätzen aus der Theaterpädagogik und aus dem Unterrichtsfach Darstellendes Spiel wird Theater mit Laiendarstellern auch immer wieder als Instrument genutzt, um Differenzen und Irritierendes wahrzunehmen, zuzuspitzen, öffentlich darstellbar zu machen sowie darüber in einen Dialog zu treten (vgl. Abschnitt 2.2. dieses Beitrags). Das Vermeiden vorschneller Wertungen, die dabei entstehende Bandbreite unterschiedlicher Spielformen und die Freude an einem positiven Kulturerlebnis kennzeichnen den beschriebenen theaterpädagogischen Ansatz. Eine performanzorientierte Aufführungspraxis, bei der es vor allem um die Darstellung von Brüchen und Differenzen geht, bietet darüber hinaus besondere Möglichkeiten für Differenzerfahrungen beim Spielen und beim Zuschauen. Wichtig dabei sind auch die möglichen Anschlusshandlungen in Form von Diskussionen über das Wahrgenommene. Dieser Gedanke steht bei der interkulturellen Theaterpädagogik im Vordergrund (cf. Sting 2008), wird aber auch zunehmend von VertreterInnen der Dramapädagogik ins Spiel gebracht (cf. Kessler \& Küppers 2008).

Die Dramapädagogik nimmt eine Zwischenstellung zwischen den fremdsprachendidaktischen Ansätzen einerseits und den Ansätzen aus der Theaterpädagogik und dem Unterrichtsfach ,Darstellendes Spiel' andererseits ein. Nach einer aktuellen Definition von Schewe handelt es sich dabei um eine „Bezeichnung für ganzheitlich orientierte Formen sprachlichen, literarischen und (inter)kulturellen Lernens, die aus der Begegnung zwischen den Bereichen Fremdsprachenvermittlung und Drama/Theater erwachsen" (Schewe 2007: 
10).

Kessler \& Küppers (2008: 9ff.) betonen die Potenziale von Drama im Hinblick auf kulturkontrastives Lernen mittels einer theatral hergestellten Wirklichkeit. Sie beschreiben Lernen durch Drama als besonderes Vehikel für (inter)kulturelle Lernerlebnisse und als einen methodischen Weg, um zunächst das abstrakte Konzept ,Kultur' erfahrbar zu machen. Bei der Arbeit mit Drama handle es sich zwar um die Schaffung und Analyse einer fiktiven Welt, aber dennoch um eine, die von unseren persönlichen und kulturellen Erfahrungen geprägt sei und uns zu einer Wahrnehmung von kulturellen Einflüssen auf unser Handeln zwinge. Dies ermögliche entschleunigte, kooperative Lernprozesse, „die das Rätselhafte im Allzubekannten wieder sichtbar werden lassen" (cf. ibid. 10). Hinzu komme die Annahme divergenter Rollen, die auch SchülerInnen zu bewusstseinserweiternden Einsichten über Kultur führen sollte. Die dramapädagogische Arbeitsweise sei auf die Wahrnehmung von Differenzerfahrung als Grundlage der Interaktion und Kommunikation angewiesen, denn „Wahrnehmungsunterschiede und deren Diskussion sind zentrale Bestandteile des dramatischen Arbeitens" (cf. ibid. 12). Fremdheit und Differenz erführen im Fremdsprachenunterricht eine zusätzliche Steigerung durch die fremde Sprache, diese sei neben ihrer Rolle als Gegenstand und Medium für die SchülerInnen gleichzeitig zusätzliche Ressource und „Kostümierung oder Verkleidung“ (cf. ibid. 13). Die Vermittlung einer interkulturellen Wissens- und Handlungsdimension im dramapädagogischen Unterricht beruhe laut Kessler \& Küppers (ibid.) darauf, dass sich das interkulturelle Wissen auf die individuelle ästhetische und emotionale Welt der Lernenden zurückbeziehen lasse, dass durch dramatische Konventionen authentische interkulturelle Lernsituationen geschaffen werden könnten und dass die SchülerInnen mit Drama immer handlungsbezogen und oft auch in konfliktträchtigen Situationen auf zukünftige interkulturelle Begegnungen vorbereitet werden könnten.

Die dramapädagogische Sichtweise stellt eine Synthese aus den unterschiedlichen Ansätzen aus der Fremdsprachendidaktik und der Theaterpädagogik dar. Das hauptsächliche Ziel besteht im Fremdsprachenerwerb und in der Persönlichkeitsbildung. Das Theaterspielen fungiert vor allem als Technik, Verfahren und Lernform.

\subsection{Einfühlung vs. Differenzerfahrung}

Aus dem Vergleich der oben dargestellten Ansätze ergibt sich die Frage, wie interkulturelles Lernen mittels Theaterspiel näher beschrieben werden kann. Liegen die Lernprozesse eher im Vorgang der Einfühlung oder der Wahrnehmung von Differenz?

Dazu muss zunächst beantwortet werden, was die Fremdsprachendidaktik unter ,Einfühlung' und ,Sich-Hineinversetzen' versteht. Meint sie das äußerlich sichtbare Sich-Hineinbegeben in eine Situation, das durch Haltungen, Mimik, Gestik und eventuell Gegenstände und bestimmte Kleidung deutlich gemacht werden kann? Dies kennzeichnet die herkömmliche Arbeit mit Rollenspielen 
im Fremdsprachenunterricht, bei welcher in der Regel das möglichst fehlerfreie Bewältigen der Kommunikationssituation und der Erwerb interkulturellen Orientierungswissens im Vordergrund stehen. Einen Restaurantdialog sprachlich fehlerfrei zu präsentieren bedeutet aber noch nicht, dass man sich auch in die fremde Lebenswelt eingefühlt hat. Diese Aufgabe ist ohne starke emotionale Beteiligung und Identifikation sowie ohne Perspektivenwechsel lösbar. Es wird keine fiktive Realität hergestellt, denn die SchülerInnen handeln in der Regel als sie selbst. Sie benutzen die Zeichen (Sprache und Gegenstände) nicht als Symbol für etwas anderes, sondern als das, was sie sind: Der Stuhl ist für den Gast, das Geschirrtuch deutet den Kellner an. Das Rollenspiel will in seiner Bedeutung nicht über das tatsächlich Gesagte hinausweisen. Es bleibt im besten Fall ein nützliches Einüben in zielsprachlich vorweggenommene Verwendungssituationen, die so oder ähnlich tatsächlich geschehen könnten.

Einfühlung in fremde Rollen und Figuren erinnert hingegen entweder an das Spiel sehr junger Kinder, die beim probeweisen Ausagieren der Welt im Spiel ihre Umgebung völlig vergessen, oder an eine spezifische Art der Schauspielerausbildung, die mit den Namen des Schauspielers, Regisseurs, Theaterdirektors und Theoretikers Stanislawski verbunden ist. Dabei geht es im Kern darum, dass die Schauspieler die Bühnenfigur mit eigenem Erleben ausfüllen. Ziel ist die möglichst realistische Darstellung auf der Bühne. Diese Aufgabe erfordert ein spezielles Training mithilfe sogenannter „Psychotechniken“ (Naumann 2003: 294).

Der Dramatiker und Theatertheoretiker Brecht hingegen betonte, dass der Wert des Schauspielens für den Schauspieler und den Zuschauer eher in der Verfremdung, in der Distanz, im Aufzeigen von Unterschieden und Differenz liege und in der Ausstellung der Veränderbarkeit der sozialen Verhältnisse (cf. Naumann 2003: 53). Diese Haltung hat sich durch die Entwicklung einer performativen Aufführungspraxis auch hierzulande in der Theaterlandschaft vielerorts durchgesetzt (vgl. Fußnote 3). Nicht die perfekte Illusion, sondern die Herausstellung des Spiels, des Unwirklichen bestimmt die Aufführungen. Äußere Merkmale sind das Ausprobieren neuer Bühnenformen (z.B. Umlaufbühne, Arenabühne), das Heraustreten aus den klassischen Theatergebäuden und das Bespielen öffentlicher Plätze ${ }^{6}$, der Verzicht auf historische Kostüme, Ausstattung und zusammenhängende Geschichten, die Beteiligung von Laien auch am professionellen Theater u.v.m.

Nicht Einfühlung und Überwindung der Unterschiede, sondern Ausstellung der Differenzen sind die wichtigsten Merkmale des modernen Theaters im 21. Jahrhundert. Sting (2008) und Kessler \& Küppers (2008) erinnern daran, dass dies zu den eigentlichen Grundanliegen, Grundaufgaben sowie -fähigkeiten von Theater gehört. Demnach liegt das besondere interkulturelle Potenzial von fremdsprachlichem Theaterspiel mit Laien nicht in der Einfühlung in vermeintlich fremde Personen, Gefühlswelten und Handlungen, sondern eher

\footnotetext{
${ }^{6}$ Beispiele: Oper in einer noch nicht eröffneten U-Bahn-Station in Berlin im Sommer 2007, Oper im Museum oder Theater in der fahrenden U-Bahn, wo das Publikum nach jeder Szene Züge und Waggons durch schnelles Hinterherlaufen wechseln muss.
} 
in der Herausstellung und Zuspitzung von Unterschieden und Differenzen mittels geeigneter theatraler Mittel.

\subsection{2 Theatrale vs. reale Wirklichkeit}

Der Vergleich der Ansätze aus der Fremdsprachendidaktik und der Theaterpädagogik ergibt ebenfalls, dass in der Fremdsprachendidaktik die Vorstellung präsent ist, dass man mithilfe von Theater unmittelbar auf zielkulturelle Kommunikationssituationen vorbereiten könne (z.B. Schewe 1993; Ronke 2005). Auch an dieser „Vorbereitungsthese“ seien Zweifel angemeldet. Theatrale und reale Wirklichkeit stehen zwar in einer Beziehung oder Verbindung zueinander, und zwar dadurch, dass sie dieselben Zeichen, Sprachen, Gegenstände usw. nutzen. Trotzdem lässt sich m.E. weder mit dem einen noch mit dem anderen Bereich umfassend auf den jeweils anderen Bereich vorbereiten. Beide Kommunikationssituationen sind grundsätzlich verschieden. Der wichtigste Unterschied besteht darin, dass alle Zeichen im Theater vollkommen neue und andere Bedeutungen annehmen können als in der Realität (vgl. Abb. 1) sowie dass die theatralen Zeichen untereinander ausgetauscht werden können (vgl. Abb. 2).
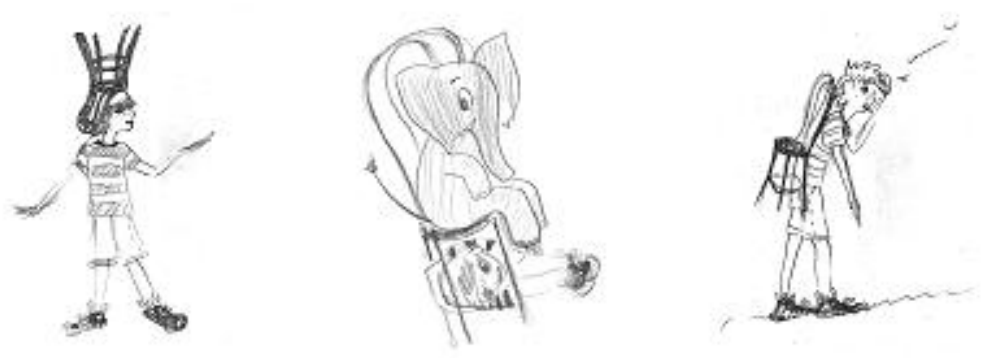

(GKatja Sittig

Abbildung 1: Potenzielle Bedeutung von Stühlen als theatrale Zeichen (ein Hut, ein Gefängnis, ein Rucksack)

Kessler \& Küppers (2008) sowie Sting (2008) weisen darauf hin, dass gerade das vom Alltag Abweichende unser Interesse weckt und unsere Aufmerksamkeit fesselt (also nicht der zielkulturelle voraussehbare Restaurantdialog, sondern 

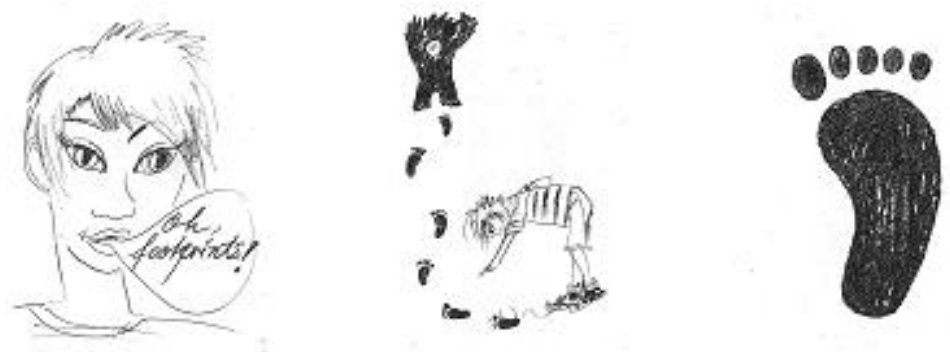

(C) Katja Sittig

Abbildung 2: Beispiel für die Austauschbarkeit theatraler Zeichen (Fußspuren können z.B. sprachlich, gestisch oder symbolisch dargestellt werden)

der ungewöhnliche Restaurantdialog, der sich so niemals abspielen würde) und unsere Wahrnehmung beeinflussen oder gar verändern kann. Anders als die Wissenschaften nutzt das Theater kein abstraktes Symbolsystem, sondern es verwendet die in der natürlichen Umgebung des Menschen vorhandenen Zeichen und Symbole, um sie auf der Bühne neu und anders ein- oder zusammenzusetzen. DieIrritation beginntbeim Besuch einerTheatervorstellung nicht erst bei der anderen Deutung bereits bekannter Sachverhalte, sondern bei der Konfrontation mit der unbekannten Verwendung bekannter Zeichen. Theater löst demnach bereits eine Irritation auf der Ebene der Wahrnehmung aus, was zu einer kognitiven Irritation und zu neuen Deutungen und Einsichten führen kann.

Im Unterrichtsfach ,Darstellendes Spiel' werden theatrale Zeichen deshalb bewusst genutzt, um eine theatrale Wirklichkeit herzustellen, die im Kontrast zu der uns umgebenden Realität steht. Ziel des ,Darstellenden Spiels' ist es nicht, in erster Linie die Wirklichkeit nachzuahmen oder zu illustrieren oder Faktenwissen darüber zu vermitteln. Es geht vielmehr darum, Zeichen aus dieser Wirklichkeit verfremdet einzusetzen und dadurch die Wahrnehmung der ZuschauerInnen zu irritieren und über diese Irritation Prozesse zur Dekodierung der theatralen Zeichen anzuregen, was wiederum zu einem Nachdenken und zu Perspektivenwechsel und Perspektivenkoordinierung in Bezug auf diese ausgestellte Realität führen kann. Eine Szene an einer Bushaltestelle, in der 
alles anders als erwartet verläuft, bringt uns stärker dazu, über erwartetes zielkulturelles Verhalten nachzudenken als eine Szene, in der interkulturelles Orientierungswissen lediglich illustriert wird. Eine wichtige Voraussetzung für die Gestaltung und die Rezeption solcher Szenen besteht darin, dass die SchülerInnen gelernt haben, theatrale Zeichen bewusst und wirkungsvoll einzusetzen.

Es lässt sich zusammenfassen, dass ein besonderes interkulturelles Potenzial von Theaterspiel im Fremdsprachenunterricht nicht unbedingt in der Illustration oder realitätsnahen Nachahmung zielsprachlicher Kommunikationssituationen besteht, sondern vor allem in der für die Zuschauenden wahrnehmungsfördernden Herstellung einer fiktiven Realität.

\section{Interkulturelles Lernen im bilingualen Sachfachunterricht ,Darstellendes Spiel“}

Die oben herausgearbeiteten besonderen Potenziale interkulturellen Lernens, die sich mit der Herstellung einer threatralen Realität mittels geeigneter theatraler Mittel und mit der Wahrnehmung und Reflexion von Differenzen beschreiben lassen, gelten gerade auch für die bilinguale Variante des Unterrichts im Fach ,Darstellendes Spiel‘. Mehr noch: Das Unterrichtsfach ,Darstellendes Spiel' bietet erst die zeitlichen und strukturellen Voraussetzungen, um mit den SchülerInnen nicht nur an der Sprache, sondern auch an den anderen theatralen Zeichen $\mathrm{zu}$ arbeiten und die Bedingungen für wirkungsvolles Theaterspiel zu erlernen. Bilingualer Sachfachunterricht ,Darstellendes Spiel bedeutet Unterricht im Fach ,Darstellendes Spiel' unter Einsatz von mindestens zwei Sprachen. Dabei kann es sich um Schulfremdsprache(n), um die Umgebungssprache(n) und die Herkunftssprache(n) der SchülerInnen handeln. Die zum Einsatz kommenden Sprachen fungieren im Unterricht hauptsächlich als Medium, als Gegenstand und als theatrales Zeichen.

Stellvertretend für die Rahmenrichtlinien für das Schulfach Darstellendes Spiel der übrigen Bundesländer soll der Berliner Rahmenlehrplan hier Auskunft über die hauptsächlich im Unterricht dieses Fachs zu entwickelnden Kompetenzen geben. Laut Berliner Rahmenlehrplan (cf. Senatsverwaltung 2006a: 12; Senatsverwaltung 2006b: 10) sollen die SchülerInnen im muttersprachlichen Unterrichtsfach ,Darstellendes Spiel ' Theater verstehen, gestalten, reflektieren, daran teilhaben und dabei theaterästhetische Handlungskompetenz entwickeln. Diese Entwicklung vollzieht sich im Fach vor allem über das eigene Spielhandeln. Es geht nicht um eine vorbereitende Schauspielerausbildung. Vielmehr sollen die SchülerInnen Erfahrungen mit dem Medium ,Theater' sammeln und zu stimmigen Darstellungen kommen. Was bedeutet das? Sie sollen mit den ihnen auch als Laien zur Verfügung stehenden Mitteln das zum Ausdruck bringen können, was sie zum Ausdruck bringen wollen. Eine wichtige Rolle spielen dabei theoretische und praktische Kenntnisse der theatralen Mittel und der notwendigen Bedingungen zur Erzeugung einer theatralen Realität. Der 
Unterricht wird in Projektform gestaltet. Als Textvorlagen eignen sich alle Texte, die das Interesse der SchülerInnen wecken, insbesondere kurze literarische oder nicht-literarische Texte, Adaptionen von professionellen Theaterbühnenstücken und Eigenproduktionen.

Wird der Unterricht in mehr als einer Sprache durchgeführt, verlagert sich der Schwerpunkt in Richtung Rezeption, Produktion und Reflexion fremdsprachigen Theaters sowie der Entwicklung der entsprechenden Theaterfachsprache. Die aus dem muttersprachlichen Unterricht im Fach ,Darstellendes Spiel ' bekannte zentrale theaterpädagogische Handlungskompetenz wird im bilingualen Unterricht um eine interkulturelle Komponente erweitert.

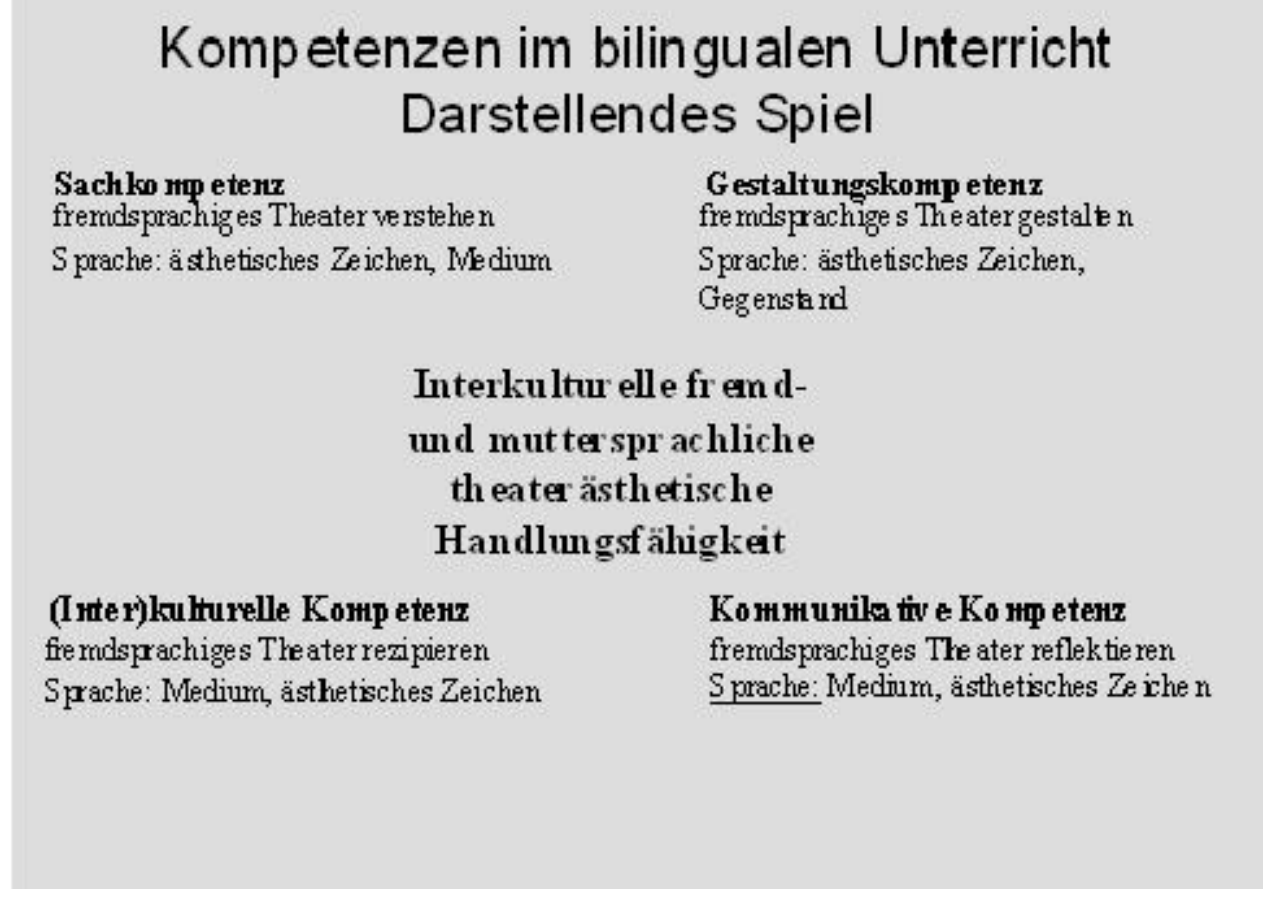

Abbildung 3: Kompetenzen im bilingualen Unterricht ,Darstellendes Spiel‘ (entwickelt nach Senatsverwaltung 2006a: 12)

Durch die Einbeziehung von mindestens zwei Sprachen folgt der bilinguale Unterricht ,Darstellendes Spiel' einem integrierten Ansatz von bilingualem Unterricht (zur Begründung dieses Ansatzes cf. Zydatiß 2002). Dieser Ansatz geht davon aus, dass jeder Sachfachunterricht letztendlich auch Sprachunterricht ist, denn mit den Konzepten müssen schließlich auch die Fachbegriffe und die damit verbundenen Bedeutungen erlernt werden. Im Zusammenhang mit fremdsprachlichem Theaterspiel nimmt die verbale Sprache eine besondere Stellung ein: Sie kann als Gegenstand, Medium und als ästhetisches Zeichen fungieren. Hier wird der aus der Dramapädagogik bekannte Umstand genutzt, dass das theatrale Mittel Fremdsprache ein Mittel der Verfremdung sein kann. Die fremde Sprache bietet den DarstellerInnen Schutz in einer fremden Rolle 
und markiert sehr deutlich den Unterschied zwischen DarstellerIn und Figur. Dadurch lädt die Fremdsprache dazu ein, höhere sprachliche Risiken einzugehen. Da die Fremdsprache häufig auch den ZuschauerInnen fremd ist, kann sie - entsprechend theatral eingesetzt - das Irritationspotenzial einer performance erhöhen (cf. Kessler \& Küppers 2008). Im bilingualen Unterricht wird die Rolle der Sprache als theatrales Zeichen gestärkt, denn der Unterricht zielt auf die Entwicklung einer fremd- und muttersprachlichen theaterästhetischen Handlungskompetenz ab.

\section{Rolle und Funktion von Sprache im Unterricht}

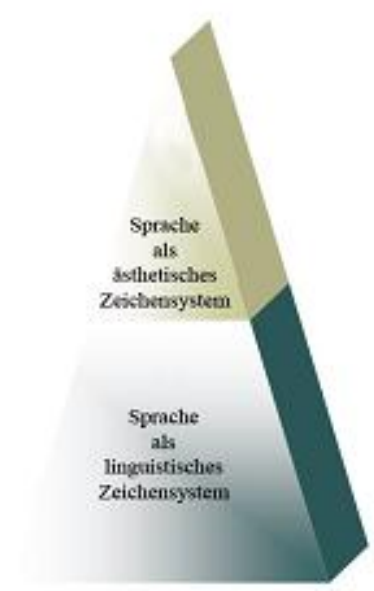

Englischunterricht

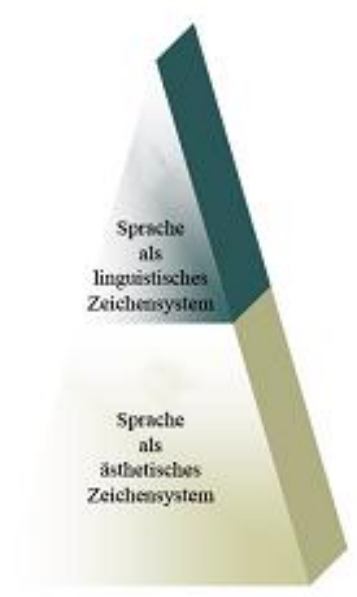

Bilingualer

Sachfachuntericht

Darstellendes Spiel

Abbildung 4: Rolle der ästhetischen Sprache im Unterricht im Vergleich

Durch das Verständnis von Sprache als theatrales Zeichen entsteht im bilingualen Unterricht ,Darstellendes Spiel' Raum für eine Lernersprache, die als poetische Sprache und damit als ästhetisches Produkt angesehen werden kann (cf. Huber 2003 zur Lernersprache als poetischer Sprache). Sprache als theatrales Zeichen sprengt die Grenzen von Einzelsprachen. Daraus ergibt sich die Möglichkeit, weitere Nationalsprachen, aber auch Phantasiesprachen und Codeswitching in den Unterricht mit einzubeziehen.

Die besondere reflexive Bildungsqualität im bilingualen Sachfach ,Darstellendes Spiel` lässt sich mit der Möglichkeit der Differenzerfahrung auf der Wahrnehmungsebene beschreiben, die zunächst auf die Verbalsprache verzichten kann. Darüber hinaus lenkt der bilinguale Unterricht ,Darstellendes Spiel die Aufmerksamkeit darauf, dass wirkungsvolles Theaterspiel auf dem Einsatz grundlegenden handwerklichen Könnens zur Schaffung einer fiktiven Realität beruht. Die SchülerInnen entwickeln sich zu kompetenten ProduzentInnen 
und NutzerInnen theatraler Zeichen. Indem der Unterricht die SchülerInnen im Umgang mit theatralen Zeichen schult, leistet er einen wichtigen Beitrag zur Medienerziehung in einer stark medial beeinflussten Umwelt, die durch sich rasant entwickelnde Zeichen und Codes gekennzeichnet ist. Diese theaterästhetische Handlungskompetenz erwerben SchülerInnen im Rahmen des bilingualen Unterrichts integriert mit den sprachlichen Kompetenzen. Damit eröffnen sich auch Möglichkeiten zur Teilnahme am internationalen, häufig von der englischen Sprache dominierten Theaterdiskurs.

\section{Fazit}

Das unverwechselbare Potenzial des interkulturellen Lernens im bilingualen Unterricht ,Darstellendes Spiel' besteht darin, dass die SchülerInnen innerhalb eines eigens dafür eingerichteten Unterrichtsfachs, eines Moduls oder eines Projekts zur Herstellung einer fiktiven theatralen Realität, zur Wahrnehmung theaterästhetischer Besonderheiten sowie zu einem nicht auf die Muttersprache beschränkten Austausch über das Wahrgenommene und die erlebten Differenzerfahrungen befähigt werden. In diesem Unterricht wird gleichzeitig an der Entwicklung der sprachlichen und der theatralen Handlungskompetenz gearbeitet. Sprache wird von Anfang an zwar als ein wichtiges, aber nicht unbedingt als das wichtigste theatrale Zeichen angesehen (für ein praktisches Beispiel cf. Wedel 2008 und Wedel in Vorbereitung).

Der bilinguale Unterricht ,Darstellendes Spiel' kann als konsequente Weiterentwicklung drama- und theaterpädagogischer Ansätze verstanden werden. Im Gegensatz zu bisher existierenden Ansätzen geht es in diesem Unterrichtsfach nicht vorrangig um den Einsatz des Theaterspielens zum Zwecke des Fremdsprachenerwerbs und der Persönlichkeitsbildung. Aber auch die theaterästhetische Seite beansprucht keine führende Rolle. Es geht im Kern um die integrierte Entwicklung von theaterästhetischen und fremdsprachigen Handlungskompetenzen. Eine wichtige Voraussetzung dafür ist, dass das Theaterspiel als eine besondere Form ästhetischer Kommunikation ernst genommen wird und die SchülerInnen durch das Kennenlernen und Experimentieren mit theatralen Zeichen auf diese Form vorbereitet werden.

Die bisherigen fremdsprachendidaktischen Ansätze haben eher weniger berücksichtigt, dass in der Arbeit an einer Bühnenpräsentation, in der Auseinandersetzung um die einzusetzenden theatralen Zeichen und deren Wirkung, das eigentliche große Potenzial fremdsprachigen Theaterspiels im Bereich des interkulturellen Lernens liegt. Indem die SchülerInnen lernen, auf der Bühne mit Hilfe bekannter Zeichen (Mimik, Gestik, Requisiten, Bühnenbild usw.) eine unbekannte und zuweilen irritierende Welt zu erschaffen, erleben sie den Prozess der (willkürlichen) Zuschreibung von Bedeutungen am eigenen Leib. Die eigentliche und ursprüngliche Aufgabe und Macht des Theaters besteht ja gerade darin, alles auf den Kopf und in Frage zu stellen, also im weitesten Sinne interkulturell zu bilden. Diese Prozesse brauchen Zeit und Raum, die das bilinguale Unterrichtsfach ,Darstellendes Spiel' bietet. 
Literatur -

Auernheimer, Georg (2003): Einführung in die interkulturelle Pädagogik. 3., neu bearbeitete und erweiterte Auflage. Darmstadt: Wissenschaftliche Buchgesellschaft.

Bonnet, Andreas; Breidbach, Stephan (2007): Reflexion inszenierbar machen. Die Bedeutung der Bildungsgangforschung für die Fremdsprachendidaktik. In: Decke-Cornill, Helene; Hu, Adelheid; Meyer, Meinert A. (Hrsg.): Sprachen lernen und lehren. Die Perspektive der Bildungsgangforschung. Opladen: Barbara Buderich, 253-272.

Caspari, Daniela (2000): Kreative Textarbeit als Beitrag zum Fremdverstehen. In: Fremdsprachenunterricht 44/53, 81-86.

Graham, Rob (1999): Theater. München u.a.: Prestel.

Hallet, Wolfgang (2005): Bilingualer Unterricht. Idee, Formen, Modelle. In: Der Fremdsprachliche Unterricht Englisch 78/39, 12.

Hoffmann, Klaus ; Klose, Rainer (Hg.) (2008): Theater interkulturell. Theaterarbeit mit Kindern und Jugendlichen. Milow/Strasburg: Schibri.

Huber, Ruth (2003): Im Haus der Sprache wohnen. Wahrnehmung und Theater im Fremdsprachenunterricht. Tübingen: Niemeyer.

Kessler, Benedikt; Küppers, Almut (2008): A Shared Mission. Dramapädagogik, interkulturelle Kompetenz und holistisches Fremdsprachenlernen. In: Scenario 2, 1-22. http:/scenario.ucc.ie

Naumann, Gabriele (2003a): Brecht, Bertolt. In: Koch, Gerd; Streisand, Marianne (Hrsg.): Wörterbuch der Theaterpädagogik. Milow [u.a.]: Schibri Verlag, 53.

Naumann, Gabriele (2003b): Stanislawski, Konstantin Sergejewitsch. In: Koch, Gerd; Streisand, Marianne (Hrsg.): Wörterbuch der Theaterpädagogik. Milow [u.a.]: Schibri Verlag, 294

Nünning, Ansgar; Surkamp, Carola (2006): Englische Literatur unterrichten. Grundlagen und Methoden. Seelze-Velber: Klett-Kallmeyer.

Raddatz, Volker (2002): Vom Wissen zum Handeln. Fremdsprachendidaktik als Mittler zwischen Theorie und Praxis. In: Neveling, Ch. (Hrsg.): Perspektiven für die zukünftige Fremdsprachendidaktik. Tübingen: Narr, 123-136.

Rademacher, Norbert (2003): Arbeitsfelder der Theaterpädagogik. In: Koch, Gerd; Streisand, Marianne (Hrsg.): Wörterbuch der Theaterpädagogik. Milow [u.a.]: Schibri, 29-30. 
Regus, Christine (2009): Interkulturelles Theater zu Beginn des 21. Jahrhunderts. Ästhetik - Politik - Postkolonialismus. Transcript: Bielefeld.

Roche, Jörg (2001): Interkulturelle Sprachdidaktik. Tübingen: Narr.

Ronke, Astrid (2005): Wozu all das Theater? Drama and Theater as a Method for Foreign Language Teaching and Learning in Higher Education in the United States. http://edocs.tu-berlin.de/diss/2005/ronke_astrid.htm.

Schewe, Manfred (1993): Fremdsprache inszenieren. Zur Fundierung einer dramapädagogischen Lehr- und Lernpraxis. Oldenburg: Zentrum für pädagogische Berufspraxis der Carl von Ossietzky Universität Oldenburg.

Schewe, Manfred (2007): Drama und Theater in der Fremd- und Zweitsprachenlehre: Blick zurück nach vorn. In: Scenario 1 (2007), http: //epu.ucc.ie/scenario/2007/01/schewe/08, 1-15.

Senatsverwaltung für Bildung, Jugend und Sport Berlin (2005): Rahmenlehrplan für die Grundschule und die Sekundarstufe I. Englisch. 1. und 2. Fremdsprache. Klasse 3-10. Grundschule, Hauptschule, Realschule, Gesamtschule, Gymnasium. Entwurfsfassung vom 26.08.2005.

Senatsverwaltung für Bildung, Jugend und Sport Berlin (2005/2006): Curriculare Vorgaben für die gymnasiale Oberstufe der Gymnasien, der Gesamtschulen mit gymnasialer Oberstufe, der Beruflichen Gymnasien, der Kollegs, der Abendgymnasien. Englisch. Gültig ab dem Schuljahr $2005 / 2006$.

Senatsverwaltung für Bildung, Jugend und Sport Berlin (2006a): Rahmenlehrplan für die gymnasiale Oberstufe. Gymnasien, Gesamtschulen mit gymnasialer Oberstufe, Berufliches Gymnasium, Kollegs, Abendgymnasium. Darstellendes Spiel. www .berlin.de/sen/bildung/schulorganisation/ lerhplaene/.

Senatsverwaltung für Bildung, Jugend und Sport Berlin (2006b): Rahmenlehrplan für die Sekundarstufe I. Jahrgangsstufe 7-10 Gesamtschule. Darstellendes Spiel. Wahlpflichtfach.

Sting, Wolfgang (2008): Interkulturell und performativ - Theater und Lernen. Dimensionen interkultureller Bildung im Theaterspielen. In: Jurké, Volker; Linck, Dieter; Reiss, Joachim (Hrsg.): Zukunft Schultheater. Das Fach in der Bildungsdebatte. Hamburg: Edition Körber-Stiftung, 87-94.

Wedel, Heike (2008): Warming Up and Cooling Down. Zu einer vernachlässigten Dimension bei der Arbeit mit dramatischen Formen. In: Ahrens, Rüdiger; Eisenmann, Maria; Merkl, Matthias (Hrsg.): Moderne Dramendidaktik für den Englischunterricht. Heidelberg: Winter, 471-492. 
Wedel, Heike (in Vorbereitung): Bilingualer Unterricht Darstellendes Spiel als interkulturelles Sprachspiel. In: Küppers, Almut; Schmidt, Torben; Walter, Maik (Hrsg.): Inszenierungen im Fremdsprachenunterricht: Grundlagen, Formen, Perspektiven. Braunschweig: Schroedel/Diesterweg (voraussichtlich 2011)

Werner, Bettina (2007): Entwicklungen und aktuelle Zahlen bilingualen Unterrichts in Deutschland und Berlin. In: Caspari, Daniela; Hallet, Wolfgang; Wegner, Anke; Zydatiß, W. (Hrsg.): Bilingualer Unterricht macht Schule. Frankfurt a.M.: Lang, 19-28.

Worsch, Michael (1996): Theaterpädagogik. In: Hierdeis, Helmart; Hug, Theo (Hrsg.): Taschenbuch der Pädagogik. Band 4. 4. vollst. überarb. und erweiterte Auflage. Baltmansweiler: Schneider-Verlag Hohengehren, 1455-1463.

Zydatiß, Wolfgang (2002): Konzeptuelle Grundlagen einer eigenständigen Didaktik des bilingualen Sachfachunterrichts. Forschungsstand und Forschungsprogramm. In: Breidbach, S.; Bach, G.; Wolff, D. (Hrsg): Bilingualer Sachfachunterricht. Frankfurt a.M.: Lang, 31-61. 